\title{
On the Ergodic Rate for Compute-and-Forward
}

\author{
Amin Sakzad, Emanuele Viterbo, Yi Hong \\ Dept. Elec. \& Comp. Sys. \\ Monash University, Australia \\ amin.sakzad, emanuele.viterbo@monash.edu \\ yi.hong@monash.edu
}

\author{
Joseph Boutros \\ Dept. Electrical Engineering, \\ Texas A\&M University at Qatar \\ boutrosetamu.edu
}

\begin{abstract}
A key issue in compute-and-forward for physical layer network coding scheme is to determine a good function of the received messages to be reliably estimated at the relay nodes. We show that this optimization problem can be viewed as the problem of finding the closest point of $\mathbb{Z}[i]^{n}$ to a line in the $n$-dimensional complex Euclidean space, within a bounded region around the origin. We then use the complex version of the LLL lattice basis reduction (CLLL) algorithm to provide a reduced complexity suboptimal solution as well as an upper bound to the minimum distance of the lattice point from the line. Using this bound we are able to find a lower bound to the ergodic rate and a union bound estimate on the error performance of a lattice constellation used for lattice network coding. We compare performance of the CLLL with a more complex iterative optimization method as well as with a simple quantized search. Simulations show how CLLL can trade some performance for a lower complexity.
\end{abstract}

Index Terms-Ergodic rate, compute-and-forward, CLLL algorithm, quantized error, successive refinement.

\section{INTRODUCTION AND MOTIVATION}

Suppose that a complex integer lattice $\Lambda=\mathbb{Z}[i]^{n}$ and a vector $\mathbf{h} \in \mathbb{C}^{n}$ are given. We consider the following optimization problem

$$
\min _{(\mathbf{a}, \alpha) \in \Lambda^{*} \times \mathbb{C}^{*}} Q(\mathbf{a}, \alpha)
$$

where $^{1}$

$$
Q(\mathbf{a}, \alpha) \triangleq \sigma^{2}|\alpha|^{2}+\|\alpha \mathbf{h}-\mathbf{a}\|^{2}
$$

The optimization problem in (1) has been investigated in the recent physical layer network coding schemes [1, 4, 6$10,12,15]$ and MIMO linear receivers [16]. In [4, 8], the optimal non-zero Gaussian integer vector $\mathbf{a}$ is chosen based on an exhaustive search within the ball of radius $1+\frac{\|\mathbf{h}\|^{2}}{\sigma^{2}}$ and then the optimum $\alpha$ was given to be the MMSE coefficient in order to maximize the computation rate of a real-valued AWGN network. However, it has been shown in [11] that the degree of freedom for compute and forward is less than 2 . In [12], the search for the optimum vector $\mathbf{a}$ is modeled as a shortest vector problem for a lattice $\Lambda$. However, an efficient approach to jointly find the optimum value of $(\mathbf{a}, \alpha)$ is not presented in these papers. It is clear that for small $\sigma^{2}$ the searching region is large and finding the optimal $\mathbf{a}$ is expensive in terms of computational complexity.

\footnotetext{
${ }^{1}$ For a set $X$, we let $X^{*}=X-\{0\}$.
}

In our paper, we consider the lattice reduction strategy to minimize $Q(\mathbf{a}, \alpha)$, a critical problem appeared in compute-and-forward protocol [8] and lattice network coding scheme [4]. Different from previous approaches in [4, 8, 12], we restrict our result to non-zero vector $\mathbf{a} \in \mathbb{Z}[i]^{n}$ and nonzero scalar $\alpha \in \mathbb{C}$. We summarize our contributions as follows.

- We use the complex version of the LLL lattice reduction algorithm (CLLL algorithm) [5] to jointly find $(\overline{\mathbf{a}}, \bar{\alpha})$ that minimize $Q(\mathbf{a}, \alpha)$. The algorithm also provides an upper bound on $Q(\mathbf{a}, \alpha)$. Furthermore, the CLLL algorithm enables us to find simultaneously $(\overline{\mathbf{a}}, \bar{\alpha}) \in\left(\mathbb{Z}[i]^{n}\right)^{*} \times \mathbb{Z}[i]^{*}$ with much lower complexity, when compared to the simple quantized approach.

- We define the ergodic rate for compute-and-forward protocol in physical layer network coding [8]. We derive a lower bound on the ergodic rate in terms of $(\overline{\mathbf{a}}, \bar{\alpha})$ derived from the CLLL algorithm [5]. We compare this bound with the ergodic capacity [3] of a multiple access channel (MAC-MISO upper bound). We also study the average error probability of a lattice network protocol [4] and derive a union bound estimate on the average error probability.

- We propose and compare the following three search methods to find $\mathbf{a}$ and $\alpha$ that minimize $Q(\mathbf{a}, \alpha)$. 1) The naive algorithm uses a simple quantized search over all possible complex values of $\alpha$. Then quantizing $\alpha \mathbf{h}$ yields the integer vector a. 2) The CLLL algorithm for an $(n+1)$-dimensional complex lattice is considered. In order to relax $\bar{\alpha}$ from $\mathbb{Z}[i]$ to $\mathbb{C}$, we propose a successive refinement search around the initial integer value of $\bar{\alpha}$. 3) An iterative MMSE based ([8]) quantization approach is used to refine the $\alpha$ provided by 1$)$.

The rest of the paper is organized as follows. In Section II, we review the CLLL-reduced basis of [5]. In Section III, we study the minimization problem with applications to computeand-forward protocol [8] and lattice network code design [4]. In Section IV, we present solutions to the optimization problem, which are based on the successive refinement of CLLL and an iterative MMSE-based quantization. In Section V, we show some experimental results. We conclude our results in Section VI.

Notations. Boldface letters are used for column vectors, and capital boldface letters for matrices. We let $\mathbb{Z}, \mathbb{C}, \mathbb{R}$, and 
$\mathbb{Z}[i]$ denote the ring of rational integers, the field of complex numbers, the field of real numbers, and the ring of Gaussian integers, respectively, where $i^{2}=-1$. We let $\mathbf{I}_{n}$ denote a $n \times n$ identity matrix. We let the operations $(\cdot)^{T}$ and $(\cdot)^{H}$ denote transpose and Hermitian transposition. The operations $\Re(\cdot)$ and $\Im(\cdot)$ denote the real and imaginary parts of a complex number. We let $|\cdot|$ denote the absolute value of a real number, or the modulus of a complex number. The $\|\cdot\|$ operation denotes the Euclidean norm of a vector. The Hermitian product of two vectors $\mathbf{a}$ and $\mathbf{b}$ is denoted by $\langle\mathbf{a}, \mathbf{b}\rangle \triangleq \mathbf{b}^{H} \mathbf{a}$. The set of orthogonal vectors generated by the Gram-Schmidt orthogonalization procedure are denoted by $\left\{\mathbf{b}_{1}^{G S}, \mathbf{b}_{2}^{G S}, \ldots, \mathbf{b}_{n}^{G S}\right\}$ spanning the same space of $\left\{\mathbf{b}_{1}, \mathbf{b}_{2}, \ldots, \mathbf{b}_{n}\right\}$. The operation $\mathbb{E}(\cdot)$ denotes mean of a random variable. We let $\lfloor x\rceil$ denote the closest integer to $x$.

\section{Complex Lattice Basis Reduction}

A complex lattice $\Lambda$ with basis $\left\{\mathbf{g}_{1}, \mathbf{g}_{2}, \ldots, \mathbf{g}_{n}\right\}$, where $\mathbf{g}_{k} \in \mathbb{C}^{n}$, includes points represented as a linear combination of basis vectors with Gaussian integer coefficients. Let us define the generator matrix for $\Lambda$ as the $n \times n$ complex matrix

$$
\mathbf{G} \triangleq\left[\mathbf{g}_{1}\left|\mathbf{g}_{2}\right| \cdots \mid \mathbf{g}_{n}\right] .
$$

One can express $\Lambda$ as $\left\{\mathbf{p}=\mathbf{G z} \mid \mathbf{z} \in \mathbb{Z}[i]^{n}\right\}$. In the lattice reduction, we let $\mathbf{B}=\mathbf{G} \mathbf{U}$, where $\mathbf{U}$ is an unimodular matrix. Let us define

$$
\mu_{\ell, j}=\frac{<\mathbf{b}_{\ell}, \mathbf{b}_{j}^{G S}>}{\left\|\mathbf{b}_{j}^{G S}\right\|^{2}}
$$

where $1 \leq \ell, j \leq n$. A generator matrix

$$
\mathbf{B}=\left[\mathbf{b}_{1}\left|\mathbf{b}_{2}\right| \cdots \mid \mathbf{b}_{n}\right]
$$

is said to be CLLL-reduced if the following two conditions are satisfied [5]

1)

$$
\left|\Re\left(\mu_{\ell, j}\right)\right| \leq 1 / 2 \quad\left|\Im\left(\mu_{\ell, j}\right)\right| \leq 1 / 2
$$

2)

for $1 \leq j<\ell \leq n$;

$$
\left\|\mathbf{b}_{k}^{G S}\right\|^{2} \geq\left(\delta-\left|\mu_{k, k-1}\right|^{2}\right)\left\|\mathbf{b}_{k-1}^{G S}\right\|^{2}
$$

where $1<k \leq n, \delta \in(1 / 2,1]$ is a factor selected to achieve a good quality-complexity tradeoff.

In [5], an algorithm was introduced to compute a CLLLreduced basis matrix $\mathbf{B}$ for a lattice $\Lambda$ with a given generator matrix G. Specifically, for an input matrix $\mathbf{G}$ and a factor $\delta$, the algorithm outputs the CLLL-reduced basis matrix $\mathbf{B}$ and the unimodular matrix $\mathbf{U}$ such that $\mathbf{B}=\mathbf{G} \mathbf{U}$. It was shown in [5] that the first column of $\mathbf{B}$, denoted by $\mathbf{b}_{1}$, satisfies

$$
\left\|\mathbf{b}_{1}\right\| \leq \beta^{\frac{n-1}{4}} \operatorname{vol}(\Lambda)^{\frac{1}{n}}
$$

where $\beta=1 /(\delta-1 / 2)$ and $\operatorname{vol}(\Lambda)$ is the volume of the lattice $\Lambda$. This provides an upper bound to the length of the shortest vector of a complex lattice $\Lambda$. The tightest upper bound is found for $\delta=1$, which leads to $\beta=2$.

\section{Main Results}

In this section, we first solve the optimization problem using CLLL algorithm. We then use this solution to derive a lower bound on the ergodic rate of compute-and-forward protocol for physical layer network coding. Similarly, we derive a union bound estimate on the average probability of decoding error for physical layer network coding.

\section{A. Methodology}

Let $\mathbf{h}=\left(h_{1}, \ldots, h_{n}\right)^{T} \in \mathbb{C}^{n}$ and $\mathbf{e}_{\ell}$ be the $\ell$-th unit vector in $\mathbb{C}^{n+1}$. Let us define an $(n+1)$-dimensional complex vector as

$$
\overline{\mathbf{h}}=\left(h_{1}, \ldots, h_{n}, \sigma\right)^{T}
$$

for some noise variance $\sigma^{2}$. Let $\Lambda$ be the lattice generated by

$$
\mathbf{G}=\left[\mathbf{e}_{1}\left|\mathbf{e}_{2}\right| \cdots\left|\mathbf{e}_{n}\right| \overline{\mathbf{h}}\right] .
$$

It follows that

$$
\operatorname{vol}(\Lambda)=|\operatorname{det}(\mathbf{G})|=\sigma .
$$

The $(n+1)$-dimensional CLLL-reduced basis generator matrix $\mathbf{B}=\left[\mathbf{b}_{1}\left|\mathbf{b}_{2}\right| \cdots\left|\mathbf{b}_{n}\right| \mathbf{b}_{n+1}\right]$ of $\Lambda$ can be computed using the aforementioned algorithm in [5]. For example, for the first column of $\mathbf{B}$, we can compute

$$
\left\|\mathbf{b}_{1}\right\|^{2} \leq 2^{\frac{n}{2}} \operatorname{vol}(\Lambda)^{\frac{2}{n+1}}=\sigma^{\frac{2}{n+1}} 2^{\frac{n}{2}}
$$

where

$$
\mathbf{b}_{1}=a_{1} \mathbf{e}_{1}+\cdots+a_{n} \mathbf{e}_{n}+a_{n+1} \overline{\mathbf{h}}
$$

for some Gaussian integers $a_{\ell}$ 's, $1 \leq \ell \leq n+1$. Defining $\overline{\mathbf{a}}=\left(a_{1}, \ldots, a_{n}\right)$ and taking $-a_{n+1}=\bar{\alpha}$, we obtain

$$
\begin{aligned}
\left\|\mathbf{b}_{1}\right\|^{2} & =\|\bar{\alpha} \mathbf{h}-\overline{\mathbf{a}}\|^{2}+\sigma^{2}|\bar{\alpha}|^{2} \\
& =Q(\overline{\mathbf{a}}, \bar{\alpha}) \leq \sigma^{\frac{2}{n+1}} 2^{\frac{n}{2}} .
\end{aligned}
$$

\section{B. Application}

The above optimization can be straightforwardly applied to the compute-and-forward protocol over finite rings [8, 14]. In [8], the optimization problem is in fact to maximize the computation rate $[8]$

$$
\mathcal{R}(\mathbf{h}, \mathbf{a})=\max _{\alpha \in \mathbb{C}^{*}} \log ^{+}\left(\frac{1}{\sigma^{2}|\alpha|^{2}+\|\alpha \mathbf{h}-\mathbf{a}\|^{2}}\right)
$$

where $\log ^{+}(x)=\max \{\log (x), 0\}$. Maximizing (5) is equivalent to minimizing $Q(\mathbf{a}, \alpha)$. Using (1), we define

$$
\mathcal{R}_{1}(\mathbf{h}) \triangleq \max _{(\mathbf{a}, \alpha) \in \Lambda^{*} \times \mathbb{C}^{*}} \log ^{+}\left(\frac{1}{Q(\mathbf{a}, \alpha)}\right)
$$

Then the definition of the ergodic rate is given below.

Definition 1: The ergodic rate $\mathcal{R}_{e}$ of a compute-andforward protocol is defined as

$$
\mathcal{R}_{e} \triangleq \mathbb{E}\left(\mathcal{R}_{1}(\mathbf{h})\right),
$$

where the mathematical expectation is taken over the channel coefficient vector $\mathbf{h}$.

We have the following theorem. 
Theorem 2: The ergodic rate of the compute-and-forward protocol is lower bounded by

$$
\mathcal{R}_{e} \geq \log ^{+}\left(\frac{1}{\sigma^{\frac{2}{n+1}} 2^{\frac{n}{2}}}\right)
$$

when the CLLL algorithm (discussed in Section III-A) is used to find $(\overline{\mathbf{a}}, \bar{\alpha})$, given $\mathbf{h}$.

Proof: The CLLL algorithm determines $(\overline{\mathbf{a}}, \bar{\alpha})$ accordingly for a given $\mathbf{h}$. Thus the ergodic rate is averaged over various channel coefficients $\mathbf{h}$. Moreover, since the function $g(x) \triangleq \log (1 / x)$ is a decreasing convex function for a positive number $x$, we have

$$
\begin{aligned}
\mathcal{R}_{e} & =\mathbb{E}\left(\mathcal{R}_{1}(\mathbf{h})\right) \\
& =\mathbb{E}\left(\max _{(\mathbf{a}, \alpha) \in \Lambda^{*} \times \mathbb{C}^{*}} \log ^{+}\left(\frac{1}{Q(\mathbf{a}, \alpha)}\right)\right) \\
& \geq \mathbb{E}\left(\log ^{+}\left(\frac{1}{\sigma^{2}|\bar{\alpha}|^{2}+\|\bar{\alpha}-\overline{\mathbf{a}}\|^{2}}\right)\right) \\
& \geq \log ^{+}\left(\frac{1}{\mathbb{E}\left(\sigma^{2}|\bar{\alpha}|^{2}+\|\bar{\alpha}-\overline{\mathbf{a}}\|^{2}\right)}\right) \\
& \geq \log ^{+}\left(\frac{1}{\sigma^{\frac{2}{n+1}} 2^{\frac{n}{2}}}\right) .
\end{aligned}
$$

where the first inequality follows from the fact that $(\overline{\mathbf{a}}, \bar{\alpha})$ is suboptimal. The inequality in (7) holds because of Jensen's inequality and the inequality in (8) holds because of (4) and the fact that $g(x)$ is a monotone function.

In the compute-and-forward protocol, we assume that each relaying node receives data transmitted simutaneously from $n$ users each with a single antenna. This scenario is very similar to a $n$-user MAC case with one transmit antenna for each user and one receiver antenna at the destination terminal. In this protocol, assuming that $\mathbf{x}_{\ell}$ is transmitted by the $\ell$-th user, $1 \leq \ell \leq n$, over a fading channel with coefficient $h_{\ell}$, the received signal at the relaying node is given by

$$
\mathbf{y}=\sum_{\ell=1}^{n} h_{\ell} \mathbf{x}_{\ell}+\mathcal{C N}\left(0, \sigma^{2}\right),
$$

and an upper bound on the instantaneous capacity is (MISO bound)

$$
C_{\mathbf{h}}=\log \left(1+\left(\sum_{\ell=1}^{n}\left|h_{\ell}\right|^{2}\right) / \sigma^{2}\right)
$$

Considering $h_{\ell} \sim \mathcal{C N}(0,1)$, we have the upper bound on the ergodic capacity (MISO bound)

$$
\mathbb{E}\left(C_{\mathbf{h}}\right) \triangleq \int_{0}^{\infty} \log \left(1+t / \sigma^{2}\right) e^{-t} t^{n-1} /(n-1) ! d t .
$$

In the next section we will compare the ergodic rate $\mathcal{R}_{e}$ and the ergodic capacity $\mathbb{E}\left(C_{\mathbf{h}}\right)$ numerically.

Let $\Lambda / \Lambda^{\prime}$ be a $n$-dimensional nested complex lattice constellation and $\mathbf{u}$ be the desired linear combination of messages with coefficient vector $\mathbf{a} \in \mathbb{Z}[i]^{n}$, and $\alpha$ be the scalar. Then the union bound estimate of the conditional probability of decoding error is given by [4]

$$
P_{e}(\mathbf{h}, \mathbf{a}) \approx K \exp \left(-\frac{d^{2}}{4 \sigma^{2}|\alpha|^{2}+4\|\alpha \mathbf{h}-\mathbf{a}\|^{2}}\right)
$$

where $d$ is the minimum inner coset distance and $K$ is the number of shortest vectors in the set $\Lambda-\Lambda^{\prime}$. According to [2], note that the sign $\approx$ can be replaced by the notation $\lesssim$. This means an approximate upper bound, one that becomes closer and closer to a true bound as $\sigma^{2}$ goes to zero. Minimizing $P_{e}(\mathbf{h}, \mathbf{a})$ in (10) is equivalent to minimizing $Q(\mathbf{a}, \alpha)$ by choosing the proper vector a and scalar $\alpha$. By substituting the upper bound (4) to the union bound estimate (10), we have the following result.

Theorem 3: Let $\Lambda / \Lambda^{\prime}$ be a nested lattice code and $\mathbf{u}$ be the desired linear combination of messages with coefficient vector $\overline{\mathbf{a}} \neq \mathbf{0}$ and $\bar{\alpha} \neq 0$ be the scalar both coming from CLLL algorithm. Then the union bound estimate for the average probability of decoding error is

$$
\mathbb{E}\left(P_{e}(\mathbf{h}, \overline{\mathbf{a}})\right) \lesssim K \exp \left(-\frac{d^{2}}{\sigma^{\frac{2}{n+1}} 2^{\frac{n}{2}+2}}\right) .
$$

\section{SeArching ApProaches}

In [8], for a given $\mathbf{a} \in \mathbb{Z}[i]^{n}$, the optimum value of $\alpha$ is given by

$$
\alpha_{\mathrm{MMSE}}=\frac{\mathrm{SNR} \cdot<\mathbf{h}, \mathbf{a}>}{1+\mathrm{SNR} \cdot\|\mathbf{h}\|^{2}}
$$

where $\mathrm{SNR}=\frac{1}{\sigma^{2}}$. However the problem of finding non-zero $\mathbf{a} \in \mathbb{Z}[i]^{n}$ is not well-addressed. In fact, the authors of [8] suggest to find this vector by doing a brute force searching in a bounded sphere of square radius $1+\mathrm{SNR} \cdot\|\mathbf{h}\|^{2}$.

Here, we propose and analyze the following search techniques to find the best a and $\alpha$. In the following proposed methods (except successive refinement of CLLL), we discretize $\alpha$ in its norm and phase and let the absolute value of $\alpha$ vary from 1 to a maximum value and phase of $\alpha$ vary from 0 to 89 degrees.

\section{A. Simple Quantized Search}

To obtain $\mathbf{a}=\left\{a_{\ell}\right\}, 1 \leq \ell \leq n$, we quantize $a_{\ell}=\left\lfloor\alpha h_{\ell}\right\rceil$. We then compute $Q(\mathbf{a}, \alpha)$ and select the lowest one and its corresponding a and $\alpha$. Note that in this case $\mathbf{a}$ is a function of $\alpha$. We call this method as "simple quantized" search. Specifically, this approach involves two passes: 1) We let $|\alpha|$ vary from 1 to a maximum value in integer steps and the phase of $\alpha$ vary from 0 to 89 degrees. We let $\alpha$ be the value that minimizes $Q(\mathbf{a}, \alpha)$. 2) We refine the search around this $\alpha$ in steps of 0.1 around the real and imaginary parts in order to find smaller values of $Q(\mathbf{a}, \alpha)$. Note that in this case $\mathbf{a}$ is a function of $\alpha$. 


\section{B. Successive Refinement of CLLL}

The CLLL algorithm finds the best $\alpha \in \mathbb{Z}[i]$ and $\mathbf{a} \in \mathbb{Z}[i]^{n}$ simultaneously, while previous technique performs a componentwise quantization of the vector $\alpha \mathbf{h}$ for each $\alpha$. Since the derived $\alpha$ is a Gaussian integer, we perform successive refinement to search for the best $\alpha$ in $\mathbb{C}$. In particular, we search around the best value of $\alpha$ delivered by the CLLL algorithm to find smaller values of $Q(\mathbf{a}, \alpha)$. Let $\alpha=\alpha_{r}+i \alpha_{i}$ be such value. We then compute $Q\left(\mathbf{a}, \alpha^{\prime}\right)$, where $\alpha^{\prime}=\alpha_{r}^{\prime}+i \alpha_{i}^{\prime}$, $\alpha_{r}-1<\alpha_{r}^{\prime}<\alpha_{r}+1$ and $\alpha_{i}-1<\alpha_{i}^{\prime}<\alpha_{i}+1$. The $\alpha_{i}^{\prime}$ and $\alpha_{r}^{\prime}$ are chosen in steps of $\eta=0.01$ in our simulations. If one results in smaller $Q\left(\mathbf{a}, \alpha^{\prime}\right)<Q(\mathbf{a}, \alpha)$, we replace $\alpha$ by $\alpha^{\prime}$. We refer this method as "successive refinement of CLLL".

\section{Iterative MMSE-based Quantization}

As in the first method we search through many values of $\alpha$ to find the best vector a by quantizing $\alpha \mathbf{h}$ componentwise. For each $\alpha$ we substitute the vector a into (12) to compute $\alpha_{\text {MMSE }}$. We let $\alpha=\alpha_{\text {MMSE }}$ and iterate the above procedure until we reach a maximum number of iterations. We select the best $\alpha$ and $\mathbf{a}$ that minimize $Q(\mathbf{a}, \alpha)$. This method is named "iterative MMSE-based quantization".

\section{Complexity Comparison}

Let us assume that the ergodic rate and the parameter $Q(\mathbf{a}, \alpha)$ for $M$ samples of $n$-dimensional channel vector $\mathbf{h}$ at a fixed SNR are of interest. We find the complexity of the above three proposed methods and the exhaustive search provided in $[4,8]$.

A) Since we search for all possible non-zero integer vectors where their square norms are less than $1+$ SNR $\cdot\|\mathbf{h}\|^{2}$, the complexity of the brute force search in $[4,8]$ is of order $O\left(M \cdot \mathrm{SNR}^{n}\right)$.

B) At high SNR's, the optimal $\alpha_{\text {MMSE }}$ is roughly equivalent to $\left\|\frac{\mathbf{a}}{\mathbf{h}}\right\|$. Therefore, the search space for $|\alpha|$ can be upper bounded by SNR. The best complexity for a sorting algorithm for each $\mathbf{h}$ is $O(M \cdot \mathrm{SNR} \cdot \log \mathrm{SNR})$. Hence the complexity order of simple quantized search and iterative MMSE-based quantization is at most $O\left(M \cdot \mathrm{SNR}^{2} \cdot \log \mathrm{SNR}\right)$.

C) The complexity of successive refinement of CLLL equals to the complexity of finding an $(n+$ 1)-dimensional complex reduced basis which is $O\left(M(n+1)^{2} \log (n+1)\right)$.

The complexity of successive refinement of CLLL is lower than all the other schemes, since it only varies by $n$. However the complexity of the other methods is based on SNR. We note that the highest complexity is for exhaustive optimal search presented in $[4,8]$.

\section{EXPERIMENTAL RESULTS}

We evaluate and compare the performance of the different methods for the 2-dimensional complex space (i.e., the two user case). The components of $\mathbf{h}$ are assumed to be circularlysymmetric complex Gaussian, with mean zero and unit variance, as in the Rayleigh fading channel model.

For the simple quantized search, we let $|\alpha|$ vary from 1 to 300 in integer steps and the phase of $\alpha$ vary from 0 to 89 by steps of 1 degrees. In a second pass we refine the search around $\alpha$ in steps of 0.05 to find smaller values of $Q(\mathbf{a}, \alpha)$. Similar refinement was performed for the CLLL method. For the iterative MMSE method we selected a maximum of 10 iterations.

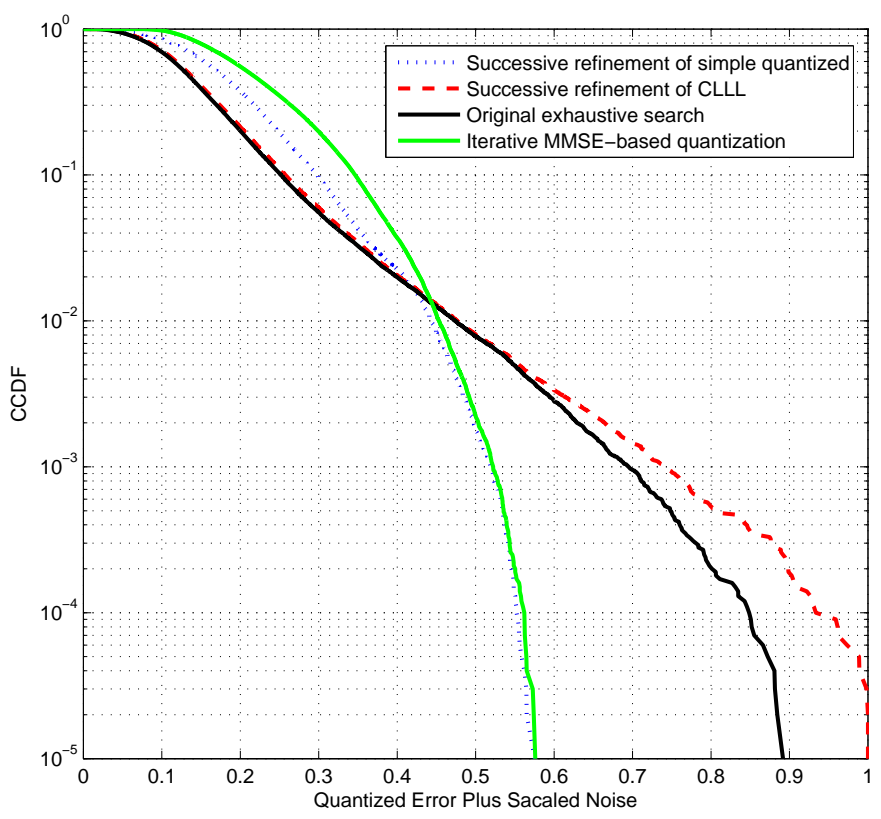

Fig. 1. Complementary cumulative distribution function for $Q(\mathbf{a}, \alpha)$ at $\mathrm{SNR}=10 \mathrm{~dB}$. The complex channel gain is circularly-symmetric Gaussian distributed $\mathcal{C N}(0,1)$.

Figs. 1 and 2 compare the complementary cumulative distribution (CCDF) of the minimum $Q(\mathbf{a}, \alpha)$ at $\mathrm{SNR}=$ 10 and $20 \mathrm{~dB}$ respectively.

Fig. 3 shows the CCDF of the minimum $Q(\mathbf{a}, \alpha)$ at $\mathrm{SNR}=$ $40 \mathrm{~dB}$ for the three methods. We observe that iterative MMSEbased quantization method exhibits the best performance at the highest complexity. The CLLL method has the lowest complexity but exhibits a slight degradation. In high SNR's the curve of CLLL algorithm via successive refinement breaks the simple quantized search one.

In Fig. 4, for different values of SNR, we plot the upper bound $\sigma^{\frac{2}{n+1}} 2^{n / 2}$ against the average values of the minimum $Q(\mathbf{a}, \alpha)$ 's for simple quantized search, successive refinement of CLLL and iterative MMSE-based quantization. The bars on curves show the standard deviation of the minimum $Q(\mathbf{a}, \alpha)$ 's for that specific SNR.

Finally, a comparison between the ergodic rates for the different strategies is given in Fig. 5. For comparison we show the capacity of a two-user MAC and the lower bound in (8). 


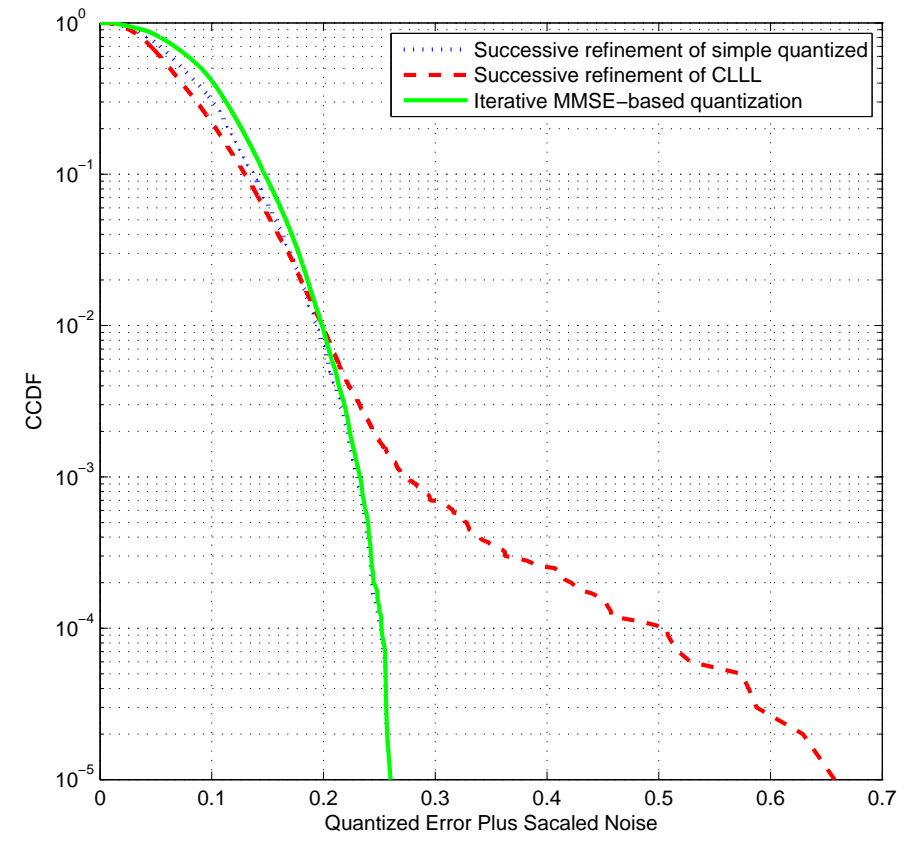

Fig. 2. Complementary cumulative distribution function for $Q(\mathbf{a}, \alpha)$ at $\mathrm{SNR}=20 \mathrm{~dB}$. The complex channel gain is circularly-symmetric Gaussian distributed $\mathcal{C N}(0,1)$. Exhaustive search is intractable at this SNR value.

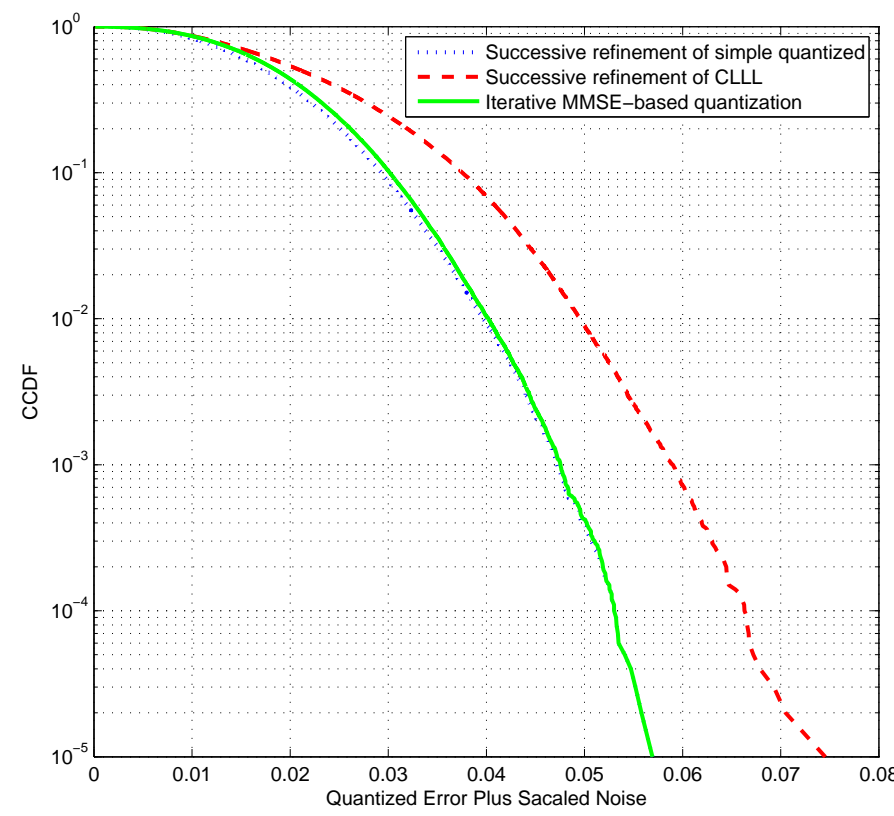

Fig. 3. Complementary cumulative distribution function for $Q(\mathbf{a}, \alpha)$ at $\mathrm{SNR}=40 \mathrm{~dB}$. The complex channel gain is circularly-symmetric Gaussian distributed $\mathcal{C N}(0,1)$. Exhaustive search is intractable at this SNR value.

\section{CONCLUSIONS}

A novel method is introduced to find the closest point of a $\mathbb{Z}[i]^{n}$ lattice to a line, within a bounded region around origin. This is also used to maximize the computation rate of a compute-and-forward protocol for a physical layer network

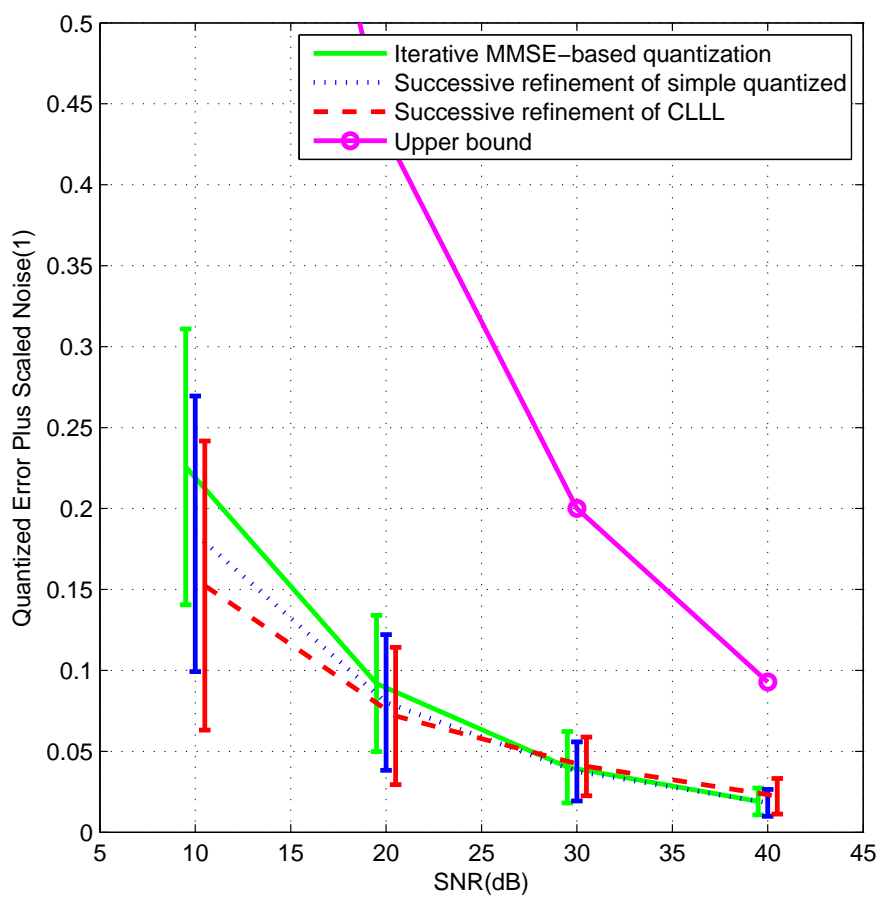

Fig. 4. Comparison between average minimum $Q(\mathbf{a}, \alpha)$ for the different approaches. The complex channel gain is circularly-symmetric Gaussian distributed $\mathcal{C N}(0,1)$. Exhaustive search is intractable at this SNR value.

coding. A lower bound on the ergodic rate and an estimation of the error performance of a lattice constellation for lattice network schemes is obtained.

We propose three methods for solving the optimization problem for $Q(\mathbf{a}, \alpha)$ : simple quantized search, successive refinement of CLLL and iterative MMSE-based quantization. The successive refinement of CLLL has two specific properties different from the simple quantized search and the iterative MMSE-based quantization. First, CLLL algorithm determines the vector $(\mathbf{a}, \alpha)$ in one step. Second, the complexity of this method is lower than the others.

Simulations are carried out to reveal the effectiveness of CLLL algorithm along with successive refinement. The result of using successive refinement of CLLL is approximately equivalent to original exhaustive search for low SNR. In particular, successive refinement of CLLL trades a little bit of performance for a reduced complexity. However the simple quantized search and iterative MMSE-based quantization method outperforms the CLLL setups.

Given the vector a, the best $\alpha$ is the one delivered by (12), which is equivalent to $\frac{\mathbf{a}}{\mathbf{h}}$ at low $\sigma^{2}$ 's. Since $\|\mathbf{a}\|$ has to large [11], if $\mathbf{h}$ be very small, then $\alpha_{\text {MMSE }}$ will be large as well. This cannot happen because $\left|\alpha_{\text {MMSE }}\right|^{2}$ has to be upper bounded by $\sigma^{2}$. This issue arises when we fix a for each $\mathbf{h}$ and differentiate $Q(\mathbf{a}, \alpha)$ to get the best $\alpha$. This is also impractical in ergodic situation because finding the best $\mathbf{a}$ is costly at low $\sigma^{2}$ 's. The experimental results suggest us to find $\alpha$ first and then put $\mathbf{a}=\lfloor\alpha \mathbf{h}\rceil$. This along with successive refinement give better performance than the above approach 


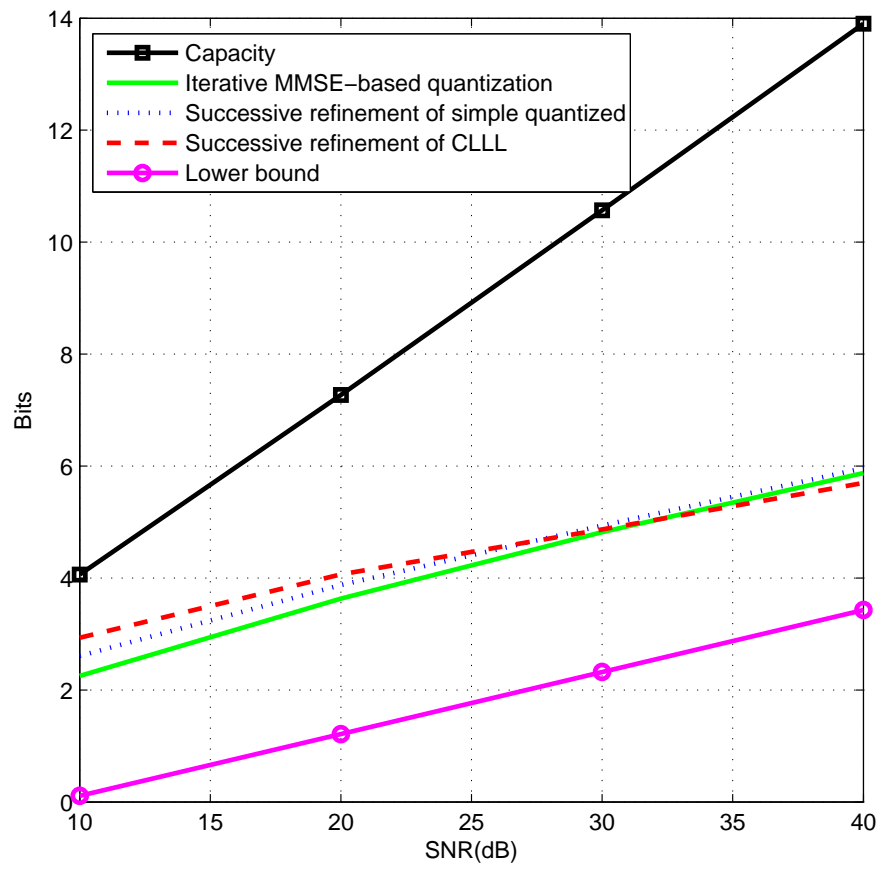

Fig. 5. Comparison between ergodic rates for the different approaches. Twouser MAC capacity and lower bound derived based on CLLL are also plotted.

at much lower complexity. Overall, we think the solution of minimizing $Q(\mathbf{a}, \alpha)$ has to be found jointly, because a and $\alpha$ are dependent in general.

In future work, we will generalize these methods to wireless network coding over finite rings [14]. We will further study good lattice constellations derived from strong infinite lattices including the ones introduced in [13].

\section{ACKNOWLEDGMENTS}

This work was performed at the Monash Software Defined Telecommunications Lab supported by the Talented Enhancement Scheme through the Monash Professorial Fellowship (MPF) program.

\section{REFERENCES}

[1] S. Avestimehr, S. Diggavi, and D. Tse, "Wireless network information flow: A deterministic approach," IEEE Trans. Inf. Theory, vol. 57, pp. 1872-1905, 2011.

[2] S. Benedetto and E. Biglieri, "Principles of Digital Transmission, with Wireless Applications" Kluwer Academics/ Plenum Publishers, 1999.

[3] E. Biglieri, J. Proakis, and S. Shamai, "Fading Channels: Information-Theoretic and Communications Aspects," IEEE Trans. Inf. Theory, vol. 44, No. 6, pp. 26192692, 1998.

[4] C. Feng, D. Silva, and F.R. Kschichang, "An Algebraic Approach to Physical-Layer Network Coding," submitted to IEEE Trans. Inf. Theory, arXiv: 1108.1695v1.

[5] Y. H. Gan, C. Ling, and W. H. Mow, "Complex Lattice Reduction Algorithm for Low-Complexity Full-Diversity
MIMO Detection," IEEE Trans. on Signal Processing, vol. 57, no. 7, pp. 2701-2710, 2009.

[6] S.-C. Liew, S. Zhang, and L. Lu, "Physical-layer network coding: Tutorial, survey, and beyond," in Phys. Commun., 2011 [Online]. Available: http://arxiv.org/abs/1105.4261.

[7] S.H. Lim, Y.-H. Kim, A. El Gamal, and S.-Y. Chung, "Noisy network coding," IEEE Trans. Inf. Theory, vol. 57, pp. 3132-3152, 2011.

[8] B. Nazer and M. Gastpar, "Compute-and-Forward: Harnessing Interference through Structured Codes", IEEE Trans. on Inf. Theory, vol. 57, no. 10, pp. 6463-6486, 2011.

[9] K. Narayanan, M.P. Wilson, and A. Sprintson, "Joint physical layer coding and network coding for bi-directional relaying," in Proc. 45th Annual Allerton Conference on Communications, Control and Computing, Monticello, IL, Sep. 2007.

[10] U. Niesen, B. Nazer, and P. Whiting, "Computation Alignment: Capacity Approximation without Noise Accumulation," submitted to IEEE Trans. Inf. Theory, arXiv: $1108.6312 \mathrm{v} 1$.

[11] U. Niesen and P. Whiting, "The Degrees of Freedom of Compute-and-Forward," to appear in IEEE Trans. Inf. Theory, arXiv:1101.2182v2.

[12] A. Osmane and J.C. Belfiore, "The Compute-andForward Protocol: Implementation and Practical Aspects," submitted to IEEE Communications Letters, arXiv:1107.0300v1.

[13] A. Sakzad, M.-R. Sadeghi, and D. Panario, "Construction of turbo lattices," in Proc. 48th Annual Allerton Conference on Communication, Control, and Computing, Allerton, Chicago, USA, pp. 14-21, 2010.

[14] E. Viterbo, Y. Hong, and J. Boutros, "Wireless Network Coding over Finite Rings," Workshop on Algebraic Structure in Network Information Theory, Banff International Research Station, Aug. 2011.

[15] M.P. Wilson, K. Narayanan, H. Pfister, and A. Sprintson, "Joint physical layer coding and network coding for bidirectional relaying," IEEE Trans. Inf. Theory, vol. 11, pp. 5641-5654, Nov. 2010.

[16] J. Zhan, B. Nazer, U. Erez, and M. Gastpar, "Integerforcing Linear Receivers," Information Theory Proceedings (ISIT), 2010 IEEE International Symposium on, pp. 1022-1026, 2010. 